\title{
SpaceX: The Unreal Reality
}

\author{
Yutika Donga \\ Student Researcher, \\ Information Technology, \\ Shree L R Tiwari College of Engineering \\ Thane, India \\ yutikadonga@gmail.com \\ Komal Champanerkar \\ Lecturer, \\ Informatio Technology, \\ Shree L R Tiwari College of Engineering \\ Thane, India \\ komal.champanerkar@slrtce.in
}

\begin{abstract}
The project aims in designing a simulation which will be operated on Android and IOS mobile comprising of gyroscope. The interactive simulation of 'SpaceX: The unreal reality', is demonstrated in our smartphone using coordinative combination of computer game and augmented reality. Here within the project the VR Box 2 is employed to look at the simulation of computer game with Gaze Interaction and Virtual Buttons are to interact in Augmented Reality. Android and iOS is that the software stack for mobile devices that has an OS, middleware and key applications. Gyroscope present within the device is employed to detect the orientation that's to augment the objects in world and consider through virtually designed world. Additionally, Unity plays a key role in developing the appliance providing vast plugins to embed $A R$ and VR technologies and also provide cross platform applicability. Augmented reality is that the mixture of video game with world, using computer generation layers to enable us an enhanced interaction with reality. This is generally done through applications, (for example, Pokemon GO), however can likewise be utilized for games, driving, and significantly more. Computer game, on the other hand, could also be a totally artificial, computer-generated simulation of a real-life experience. This typically required the use of a video game headset, like Oculus Rift or HTC Vive, so on to completely immerse the user. This project contains various user interaction by means of virtual buttons, gaze interaction and speech recognition The project eventually provides the Mixed Reality Simulation combining both AR and VR technologies and depicts the vision of Elon Musk's Mission of SpaceX to colonize Mars.
\end{abstract}

Keywords: Unity, Virtual Reality, Augmented Reality, Mixed Reality, Virtual Buttons, Gaze Interaction, Speech Recognition, Android and $\mathrm{iOS}$.

\section{INTRODUCTION}

\section{A. Augmented Reality}

An upgraded form of reality where live immediate or roundabout perspectives on actual genuine conditions are increased with superimposed PC produced pictures and perceptual data, preferably over various tangiblemodalities, including visual, hear-able, haptic, somatosensory, and olfactory over a client's perspective on this present reality, hence improving one's present impression of the real world. The overlaid sensory information can be constructive (i.e. additive to the natural environment) or destructive (i.e. concealing of the common habitat) and is spatial enrolled with the actual world such it's apparent as a vivid part of the significant climate. Augmented Reality is said to 2 largely synonymous terms: mixed reality and computer-mediated reality. Expanded reality applications are written in uncommon $3 \mathrm{D}$ projects that permit the engineer to tie movement or relevant computerized data inside the PC infection to an enlarged reality "marker" in reality. At the point when a figuring gadget's AR application or program module gets computerized data from a known marker, it starts to execute the marker's code and layer the correct picture or pictures.

\section{B. Virtual Reality}

Virtual Reality may be a computer-generated scenario that simulates a sensible experience. The vivid climate can be like this present reality to make a similar grounded in actuality or science fiction. It is a man- made climate that is made with programming and introduced to the client in such how that the client suspends conviction and acknowledges it as a genuine climate. Virtual reality is principally experienced through two of the five detects: sight and sound. The computer game Modelling Language allows the creator to specify images and therefore the rules for his or her display and interaction using textual language statements. Virtual reality can be partitioned into: The recreation of a genuine climate for preparing and schooling OR The improvement of an envisioned climate for a game or intelligent story. Current VR innovation most usually utilizes PC game headset or multi-extended conditions, some of the time along with actual conditions or props, to get practical pictures, sounds and different vibes that recreate a client's actual presence during a virtual or nonexistent climate.

\section{Mixed Reality}

Blended Reality is referenced as crossover the truth is that the converging of genuine and virtual world to gracefully new conditions and representations where physical and advanced items coincide and cooperate continuously. Mixed reality takes place not only within the physical world or the virtual world but may be a mixture of reality and computer game, encompassing both augmented reality and virtual reality via immersive technology. Mixed reality not just overlays but anchors virtual objects to the important world. In mixed reality environments, users seamlessly navigate through both the important and virtual environments at an equivalent time. Instead of residing in a completely virtual world (for example computer generated reality), virtual articles are secured into a client's certifiable space and expand their genuine climate, causing virtual connections to have all the earmarks of being "genuine". These interactions mimic our natural behavior of interaction, 
like objects getting bigger as you meet up with and therefore the changing of perspectives as you progress around an object. Mixed reality may be a blend of the planet we sleep in enhanced by technology that brings another dimension to what we are seeing, feeling, and tasting. The applications inside the occasion circle are unfathomably intriguing.

\section{PROBLEM STATEMENT}

Simulation is that the imitation of the operation of a real- world process or system. The demonstration of recreating something initially necessitates that a model be created; this model speaks to the key qualities, practices and elements of the picked physical or unique framework or cycle. The re-enactment speaks to the activity of the framework after some time. Reproduction is utilized in numerous unique situations, similar to re-enactment of innovation for execution streamlining, wellbeing designing, testing, preparing, training, and computer games. Now-a-day creating a simulation using upcoming technologies and enhancing its potential is necessary. The advancements like Augmented Reality for example is the incorporation of advanced data with the client's current circumstance progressively utilizing the overall climate and overlaying new data on top of it. And video game which may be a man-made environment that's created with software and presented to the user in such how that the user suspends belief and accepts it as a real environment. Augmented Reality and Virtual Reality provides a better understanding of real world system by fusing them into a combination of known as Mixed Reality. Blended Reality is that the converging of genuine and virtual world to give new conditions and perceptions where physical and advanced articles exist together and

interface continuously. Blended reality happens not just inside the actual world or the virtual world yet might be a blend of the real world and computer generated reality, incorporating both expanded reality and augmented reality by means of vivid innovation. Thus, using a fusion of Virtual Reality and Augmented Reality (known as Mixed reality) we aim to create a real-world scenario that depicts the Mission of SpaceX by Elon Musk to colonise Mars and transform it to create a multi planet species. This Simulation makes user experience the whole mission and vision how Elon Musk thought of having it in future.

\section{LITERATURE REVIEW}

The Literature survey here outlines preceding researches on Virtual reality, the algorithms and graphs used by them. The writings we present here is the work of many pertinent papers explored by us so by collecting the combination of keywords and snowballing we have improvised our project. The literature review makes us contemplate and understand the earlier innovations related to the project.

1. The main purpose of this paper is to build an end-toend system architecture for the VR space that is based on the real world along with the element technologies that constitute the proposed system. This paper basically focussed on how videos and images can be used to create virtual reality world and also described many methods through which we can achieve this. VR world can be reconstructed from images/videos that are captured and stored. Various methods can be used for reconstructing the virtual view but the method that is described and is useful for our project is image stitching method. If the stored images are numerous and various, it can provide a wider range of viewpoints.

2. This paper basically explains the importance of VR and AR. The game industry is a major adopter of VR and its sibling AR. Human expressions, reporting, the military, NASA, medication, and training are additionally grasping the capability of AR and VR for articulation, correspondence, joint effort, and learning. This paper gave us the idea of creating a virtual reality world of space. Most of the people cant get the real experience of space. So through this project we can help them to get the real experience of space. Gesture-recognition systems and alternative controllers dependent on computer vision, camera systems will complete the immersive experience.

3. A virtual reality system is allowing the user to search and interact with a three dimensional virtual or artificial environment created by the designer. In the virtual world, the user can do all things almost like routine as throwing a ball or as excellent as flying through space. Bharath et al. introduced their paper on "Importance \& Applications of Virtual Reality in Engineering sector". Radharamanan et al. spoken to their paper on "A survey of computer game technologies, it's applications and limitations". From this paper we extracted the working principle of VR technology: It first tracks the physical movements in the real world, then a computer redraws the virtual world to reflect those movements. The updated virtual world is shipped to the out-put. The output is shipped back to a head mounted display. Consequently, the client feels "drenched" inside the virtual world as though they're inside the virtual world itself as all they will watch is their delivered developments in virtual climate.

4. Redirected Walking (RDW) could also be a set of video game (VR) locomotion techniques for immersive virtual environments (IVE) that allows humans to steer on paths within the planet, which may vary from the paths they perceive within the virtual environment (VE). Since RDW was first presented, there is still no standard answer for limitless strolling in a confined following space without interferences. Steering algorithms and path prediction methods might be improved using machine learning algorithms. While actualizing RDW with ebb and flow increases, two things had the chance to be given or expected: (I) the objective of the client inside the VE, and (ii) the objective of the redirection in reality.

5. This paper represent a captured indoor environment into 3D content ready for AR/VR applications, with full geometry, texture, and lighting information. Examining and obtaining a 3D indoor climate experiences complex impediments and misalignment blunders. The reproduction acquired from a RGB-D scanner contains openings in calculation and ghosting in surface. These are easily noticeable and 
can't be considered as visually compelling VR content without further processing. Given an underlying $3 \mathrm{D}$ recreation caught by a RGB-D sensor, we use planes not exclusively to speak to the climate mathematically yet additionally to unwind an opposite delivering issue thinking about surface and light. The complex process of shape inference and intrinsic imaging is greatly simplified with the assistance of detected planes and yet produces a sensible 3D indoor environment.

\section{PROPOSED SYSTEM}

We propose a Mixed Reality system which blends a combination of both Virtual Reality and Augmented Reality. It depicts the SpaceX Mission to Mars by Elon Musk. It also interprets the concept of Virtual and Real World intertwined, providing an interaction with and manipulation with both virtual and physical environment. The user experiences the journey passing through a city to space station and from space station to actual rocket launching site. The user has two options either to choose Augmented Reality or Virtual Reality. While using Augmented Reality the user is provided by Virtual Buttons intercepted in Real World and While using Virtual Reality the user is provided by Gaze Interaction. After rocket launch the user experiences a space journey full thrill experiencing user with solar system, planets, star field, debris, asteroids, satellites and space station.

And finally, the rocket reaches planet Mars and landed on its surface. After successful landing the user has again two options to choose Augmented Reality and Virtual Reality. Here the total vision of Elon Musk Mission of SpaceX is depicted by Mixed Reality. The user has various button panel to deploy and colonise mars step by step.

First the Astronauts and Space Vehicle like hovers and space cars are deployed and then the whole infrastructure including space station and domes with high tech facilities. The Mission also aims at deploying water (H2O), Atmosphere $(\mathrm{CO} 2, \mathrm{O} 2)$ and plants on Mars to transform it completely into another Earth for Humans to stay and create spacefaring civilization and a multiplanet species.

\section{ADVANTAGES}

1. It creates a realistic world and enables user to explore places and user can experiment with an artificial environment.

2. User gets a better understanding of modelling the real-world systems and play around with them.
3. It provides a realistic experience by interactive gaze view and virtual system.

4. Provides Outstanding Visualizations that aren't possible in the traditional methods and creates interest among the users.

5. This simulation provides instant results and analyse outcomes. This will eventually improve execution and empower refined criticism to students during explicit assignments. It also increases efficiency and greater awareness or judgements of one's abilities.

6. The project provides a user-friendly GUI where the user doesn't need to use much thinking skill set.

7. The project also provides a website with detailed information about AR VR and MR. It provides a user manual and downloads of different version of our application.

\section{CONCLUSION}

In this project we have proposed to develop a powerful and useful features of human interaction, an informative and attractive user interface, website to download access to this project and explore its different versions. The feature of speech recognition allows us to make use of Artificial Intelligence. Other than enhancing our Programming, Modelling and Animation skills, we will get to learn a lot of qualities like team work, dividing and coordinating work, developing innovative ideas, etc.

\section{REFERENCES}

[1] Eike Langbehn and Frank Steincke, "Redirected Walking in Virtual Reality", Springer March 2018.

[2] Young Min Kim, Sangwoo Ryu and Ig-Jae Kim, "Planar Abstraction and Inverse Rendering of 3D Indoor Environments", IEEE Transactions on Visualization and Computer Graphics, Vol. XX, No. X, August 2019.

[3] Dongho You, Bong-Seok Seo, Eunyoung Jeong and Dong ho kim, "Internet of Things(IoT) for Seamless Virtual Reality Space: Challenges and Perspectives", Seoul National University of Science and Technology, South Korea 2018.

[4] Ann DeMarie, "Let Us Entertain You", IEEE September 2017.

[5] Ronak Dipakkumar Gandhi and Dipam S. Patel, "Virtual Reality Opportunities and Challenges", IRJET, Volume 5, January 2018.

[6] Vladimir M. Petrović, "Artificial Intelligence and Virtual Worlds Toward Human-Level AI Agents”, IEEE 2017.

[7] João Pedro Assunção Campos and Rafael Rieder, "Procedural Content Generation using Artificial Intelligence for Unique Virtual Reality Game Experiences", 21st Symposium on Virtual and Augmented Reality (SVR), IEEE 2018. 